\title{
Scalable Low-Carbon Hydrogen Production Technology Utilizing Waste/Process Heat
}

\author{
Kamiel S. Gabriel \\ Ontario Tech University \\ Oshawa, ON, Canada \\ kamiel.gabriel@ontariotechu.ca
}

\begin{abstract}
Achieving net-zero emissions requires a radical transformation in the way we produce, supply, transform and use energy. The rapid growth of wind, solar and electric cars has shown the potential of how new clean energy technologies can bring down emissions.

Reaching net-zero emissions in 2050 would require a much more rapid deployment of low-carbon power generation. But electricity cannot decarbonize entire economies alone. Hydrogen forms a bridge between the power sector and industries. In fact, Hydrogen and CCUS account for about half of cumulative emissions reductions in the steel, cement and chemicals sectors. In the trucking, shipping and aviation sectors, use of alternative fuels - hydrogen, synthetic fuels and biofuels - ranges between $55 \%$ and $80 \%$.

Earlier analysis of future energy pathways shows that it is technically possible to achieve improved energy access, air quality, and energy security simultaneously while avoiding dangerous climate change. In fact, a number of alternative combinations of resources, technologies, and policies are found capable of attaining these objectives.

Although a successful transformation is found to be technically possible, it will require the rapid introduction of policies and fundamental political changes toward concerted and coordinated efforts to integrate global concerns, such as climate change, into local and national policy priorities such as health and pollution, energy access, and energy security.
\end{abstract}

